\title{
Low-Energy Linear Structures in Dense Oxygen: Implications for the $\epsilon$-phase
}

\author{
J. B. Neaton ${ }^{1}$ and N. W. Ashcroft ${ }^{2,3}$ \\ ${ }^{1}$ Department of Physics and Astronomy, Rutgers University, Piscataway, NJ 08854-8019 \\ ${ }^{2}$ Laboratory of Atomic and Solid State Physics and Cornell Center for Materials Research, \\ Cornell University, Ithaca, NY 14853-2501 \\ ${ }^{3}$ Cavendish Laboratory, University of Cambridge, Madingley Road, Cambridge, CB3-0HE, United Kingdom
}

(January 25, 2002)

\begin{abstract}
Using density functional theory implemented within the generalized gradient approximation, a new non-magnetic insulating ground state of solid oxygen is proposed and found to be energetically favored at pressures corresponding to the $\epsilon$-phase. The newly-predicted ground state is composed of linear herringbone-type chains of $\mathrm{O}_{2}$ molecules and has $C m c m$ symmetry (with an alternative monoclinic cell). Importantly, this phase supports IR-active zone-center phonons, and their computed frequencies are found to be in broad agreement with recent infrared absorption experiments.
\end{abstract}

PACS: 61.50.Ah, 78.30.Am

At low temperatures and ordinary pressures solid oxygen condenses into the only antiferromagnetic insulating phase known among the elemental solids. In its ground state, the well-known monoclinic $\alpha$-phase [1], the molecular spins are arranged in nearly-closed packed planes (or layers) perpendicular to the molecular axes; the axes of molecules in successive planes are collinear [1] [3] (and apparently so in all measured phases). As the temperature is increased, oxygen eventually undergoes a transition to its familiar room-temperature gaseous phase, but as the pressure is increased, its attributes depart radically from those of an ensemble of weakly-interacting molecules. In fact at pressures above $96 \mathrm{GPa}$ (at nearly 3 -fold compression), both diamond-anvil [田5] and shock [6] experiments have reported an insulator-metal transition and, at temperatures below $0.6 \mathrm{~K}$, the metallic state (the $\zeta$-phase) even exhibits superconductivity [7]. While some of the broader aspects of the electronic behavior are understood throughout this density range, the crystal structure and magnetic properties of the so-called $\epsilon$-phase, persisting as it does over the wide, intermediate pressure range of 10-96 GPa, remain unknown.

Over two decades ago, a dramatic color change was observed (from light-blue transparent to darkening red) in experiments above $10 \mathrm{GPa}$ at room temperature [8]. Detailed optical measurements [2] shortly thereafter at room temperature revealed an abrupt onset of infrared (IR) absorption in the fundamental molecular vibron, also at approximately $10 \mathrm{GPa}$. Recent low-frequency optical studies have uncovered another strong IR peak, ranging in frequency between $300-500 \mathrm{~cm}^{-1}$ from 10 to $70 \mathrm{GPa}$, but at very much lower frequencies than that of the vibron $\left[13\right.$ 115]. The formation of pairs of $\mathrm{O}_{2}$ molecules with $\mathrm{D}_{2 h}$ (rectangular) symmetry-an $\mathrm{O}_{4}$ unit-has been suggested to explain these new low-frequency modes 13, 15. Interestingly enough the sudden increase in the intensity of infrared activity in two quite different frequency bands parallels the case of dense hydrogen, where a spontaneous polarization of the charge density along the molecular bonds has been suggested to account for the stability of low-symmetry structures above $150 \mathrm{GPa}$ 16.

Although there exist several promising structures 9-14, to date $\mathrm{x}$-ray diffraction studies have been unable to completely reveal the exact positions of the molecules in the $\epsilon$-phase. Likewise a previous first-principles study recorded a premature magnetic collapse and concomitant metallization into to the $\zeta$-phase near 12 GPa [17], bypassing the $\epsilon$-phase. In this Letter we summarize results of first-principles calculations that predict a new lowenthalpy molecular arrangement in the pressure range of the $\epsilon$-phase. Beginning with a symmetrical low-density structure, we observe that it is unstable to the formation of extended herringbone-type chains of $\mathrm{O}_{2}$ molecules (instead of $\mathrm{O}_{4}$ units), strikingly similar to that suggested by Agnew et al. [11]. Non-magnetic and insulating, this newly-predicted phase is also consistent with recent infrared measurements.

We examine here the stability of the common symmetric phases of oxygen over the molar volume range 6.7$13 \mathrm{~cm}^{3} /$ mole, the lower value corresponding to pressures exceeding $100 \mathrm{GPa}$. Density functional theory (DFT) is used within the local spin-density approximation (LSDA) 18] and with gradient corrections [19]. We utilize the projector augmented-wave (PAW) method [20] as implemented within the Vienna ab initio Simulations Package (VASP) 21. Our oxygen PAW potential relegates the $1 s$ electrons to a frozen core but otherwise treats all other electrons explicitly as valence; a 60 Ry plane wave cutoff is used for all calculations. These methods provide a particularly accurate picture of the free molecule for which we correctly obtain the magnetic $(S=1)$ ground state; our calculated bond length of $1.236 \AA$, binding energy of near $6.0 \mathrm{eV}$, and molecular vibron frequency of $1550 \mathrm{~cm}^{-1}$ (within the harmonic approximation) are all slightly larger than experiment $(1.207 \AA$ ) but quite consistent with the known tendency of gradient corrections to overestimate the bond length and binding energy of $p$-bonded diatomic molecules [22]. 
A primary physical issue centers on the notably high linear dipole polarizability of the oxygen molecule. The along-axis tensor component is $15.9 a_{0}^{3}$ and the off-axis components are $8.2 a_{0}^{3}$ [23], where $a_{0}$ is the Bohr radius. These exceed the corresponding quantities in hydrogen by more than a factor of two, and the Hertzfeld criterion (for the onset of a polarization divergence) would require a compression of only about 1.8. There is a large anisotropic fluctuating-dipole (or van der Waals) attraction [24], and an accurate, effective, and fully non-local representation of these correlated fluctuations within density functional theory has proven to be a challenge 25,26]. Thus the local density approximation is not expected to be satisfactory until significant intermolecular density has accumulated, and accordingly we focus our study on volumes less than $13 \mathrm{~cm}^{3} /$ mole $(8 \mathrm{GPa})$, above which we obtain adequate agreement $( \pm 5 \mathrm{GPa})$ with the equation of state at $300 \mathrm{~K}[$ 国.

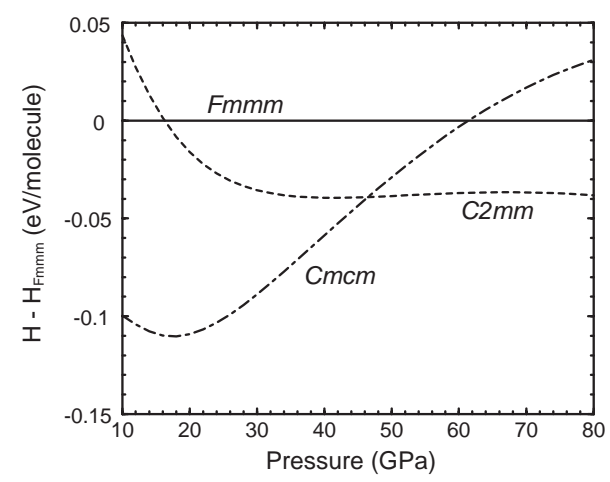

FIG. 1. Enthalpy $(H=E+p V)$ vs. pressure $p$ for selected structures of dense oxygen with respect to the $F m m m$ phase at $T=0 \mathrm{~K}$. In general agreement with a previous study [17], we observe that the $F m m m$ phase is unstable to $C 2 \mathrm{~mm}$ above $17 \mathrm{GPa}$. Convergence with respect to k-points is achieved at $1 \mathrm{meV} /$ molecule using 10x10x12 Monkhorst-Pack k-meshes for 2-molecule primitive cells; more $\mathbf{k}$-points are used for the metallic phase. Relaxations are performed until Hellmann-Feynman forces are less than $10^{-2} \mathrm{eV} / \AA$. The enthalpy $H$ was determined by fitting the energy $E$ to $\sum_{n=-2}^{2} a_{i} V^{-n / 3}$.

At room temperature and under a moderate compression of $8 \mathrm{GPa}$, the antiferromagnetic $\delta$-phase (space group Fmmm) of solid oxygen has been particularly wellcharacterized [2, 3, 8. However recent x-ray data suggests a direct $\alpha$-to- $\epsilon$ transition at low temperatures [12]. Further, IR spectra [14] and Raman [27] between 2-8 GPa and below $20 \mathrm{~K}$ appear to be inconsistent with $\mathrm{Fmmm}$ symmetry. Thus in order to examine the possibility of lower symmetry ground states that $d o$ possess IR activity at the observed frequencies and remain insulating, we released the symmetry constraints of a 4-molecule Fmmm orthorhombic cell and completely relaxed the internal coordinates and lattice parameters, all with dense $\mathbf{k}$-point sampling. This immediately resulted in a significant rearrangement of the crystal and a new, stable orthorhombic structure, possessing a two-molecule primitive cell and $\mathrm{Cmcm}$ space-group symmetry. A plot of the enthalpies as a function of pressure for all structures considered appears in Fig. 1. For comparison, we also evaluate the enthalpy of the metallic $C 2 \mathrm{~mm}$ phase, a candidate $\zeta$ phase proposed by Serra et al. [17]. Interestingly we find the $C m \mathrm{~cm}$ structure to be unstable to $C 2 \mathrm{~mm}$ at a calculated pressure of $47 \mathrm{GPa}$, resulting in an insulator-metal (IM) transition at a lower pressure than experimentally observed (96 GPa 团). Since the IM transition is associated with band gap closure, this discrepancy may result from the usual underestimate of the electronic band gap by the gradient-corrected LDSA.

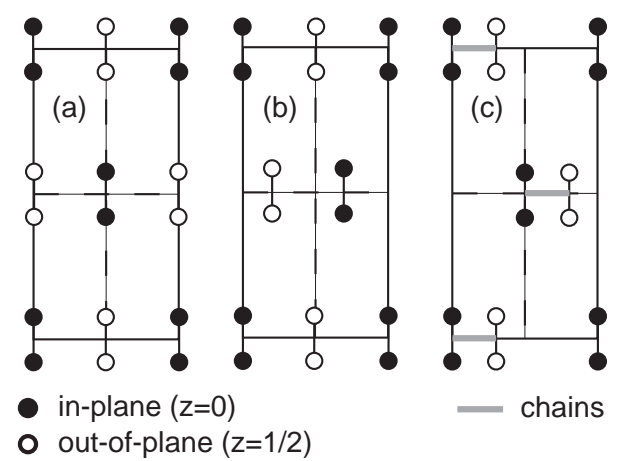

FIG. 2. Schematics of (010) ac-planes of (a) Fmmm, (b) $C 2 \mathrm{~mm}$, and (c) newly-predicted $\mathrm{Cmcm}$ phases. The molecules in white (oriented along [001]) are shifted by half a lattice vector out of the plane. The orthorhombic $\mathrm{Cmcm}$ phase (having Wyckoff positions $8(\mathrm{~g})$ ) results from an intraplanar distortion in which the center molecule moves off-site. Chains run perpendicular to the plane and are indicated by with bold gray lines. Differences in, e.g., $a$ or $c / a$ are ignored in these illustrations.

Atomic arrangements for the three phases considered here appear in Fig. 2. In the $\mathrm{Cmcm}$ structure the oxygen molecules order into symmetric herringbone-type chains along [010] (the $b$-axis) and perpendicular to the molecular bonds. They form through the shearing of adjacent (010) planes of the Fmmm structure, reducing the coordination of the molecules from four to two. An $\epsilon$-phase having monoclinic symmetry has been suggested [3, 1, 12], and in this context we note that a monoclinic primitive lattice vector $\mathbf{c}^{\prime}$ can be chosen for $\mathrm{Cmcm}$ (where $\left|\mathbf{c}^{\prime}\right|=\frac{1}{2} \sqrt{a^{2}+c^{2}}$ ). (A similar relationship exists between the $\alpha(C 2 / m)$ and $\delta(F m m m)$ phases 12 .) Nevertheless we find that distortions of its orthorhombic lattice vectors a and $\mathbf{c}$ (resulting in $P 2_{1} / c$ symmetry) do not lower the total energy [28]. The Fmmm phase is related through continuous distortion to the nearly close-packed $C 2 \mathrm{~mm}$ phase by a displacement of (001) planes in the [100] direction 29. Interestingly, the dimer length is found to decrease by about $1 \%$ with increasing pressure in this new phase, from $1.224 \AA$ at $8 \mathrm{GPa}$ to $1.209 \AA$ at $54 \mathrm{GPa}$. 
The distance between neighboring molecules along the chain is reduced from $2.081 \AA$ to $1.986 \AA$ over this range, a modest overbinding with respect to experiment, where these distances are thought to be in the range 2.2-2.5 [13]. Similarly, the $a, b$, and $c$ lattice parameters decline from 3.805 to $3.333 \AA, 2.990$ to $2.718 \AA$, and 7.034 to $6.216 \AA$, respectively, as pressure is increased from 8 to $54 \mathrm{GPa}$. Notably, $c$ diminishes slightly faster than either $a$ or $b$ with increasing pressure; experimentally, the $b$-axis is observed to decrease most rapidly 14. As the $c$-axis spacing is much larger than either $a$ or $b$, remnant van der Waals interactions (see above) may still be particularly important between adjacent (001) planes, and therefore it is here that our treatment of exchange and correlation is likely to be most inadequate.

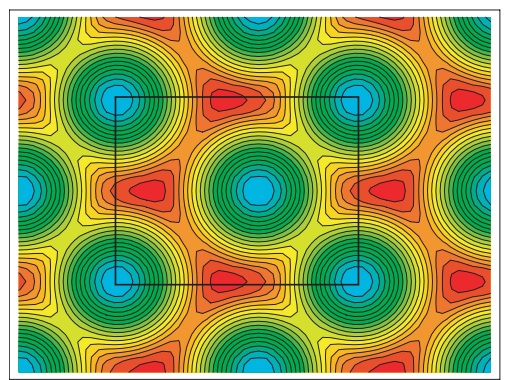

FIG. 3. Charge density slice of $\mathrm{Cmcm}$ at a calculated pressure of $20 \mathrm{GPa}\left(10.26 \mathrm{~cm}^{3} /\right.$ mole) plotted in an (001) (or $a b)$ plane passing through the molecular centers (the unit cell is outlined in black). A natural logarithmic scale is used; the highest contour (blue) are 54 times larger than the lowest (red). Since the chains are equally spaced in the cell, the development of an alternating polarization perpendicular to the molecule is evident by inspection.

Solid oxygen is robustly insulating in the $\mathrm{Cmcm}$ structure, and the calculated band gap (a considerable underestimate of the true band gap) decreases from 0.95 $\mathrm{eV}$ (direct gap) near $8 \mathrm{GPa}$ to about $0.55 \mathrm{eV}$ (indirect gap) at $\sim 55 \mathrm{GPa}$. The optical threshold of the $\epsilon$-phase has been measured to be around $3 \mathrm{eV}$ (blue) near 10 $\mathrm{GPa}$, declining to $\sim 2.0 \mathrm{eV}$ (orange-red) near $55 \mathrm{GPa}$ [5], for light polarized perpendicular to the molecular axis. These are larger than our computed direct gaps at these pressures (for the smallest, by a factor of three) but, as stated above, this is to be expected given our use of the LSDA; corrections through approximate inclusion of many-electron effects via, for example the GW approximation [30], would be of considerable interest for accurately reproducing this gap and likewise describing the onset of metallization.

The charge density is plotted in Fig. 3 in the $a b$-plane (perpendicular to [001]), and is seen to develop an asymmetry occurring perpendicular to the molecular bond but centered on each molecule, as is evident by inspection of Fig. 3, providing insight into the stability of this phase. The asymmetry may be viewed as a weakly antiferroelec- tric state, whose physical origin may in turn be understood through consideration of a mean-field argument for a dynamic lattice as given for hydrogen [16]. Thus selfconsistent Lorenz fields compensate energetic costs associated with the low-symmetry $\mathrm{Cmcm}$ structure in the intermediate pressure range below $50 \mathrm{GPa}$. Increasing intermolecular overlap at higher densities lowers kinetic and exchange energies, and the metallic $C 2 \mathrm{~mm}$ phase is thus eventually favored. Examination of the spin density indicates that oxygen is non-magnetic within this phase, consistent with other studies [13,31; total energies and forces obtained from spin- and non-spin-polarized calculations are essentially identical.

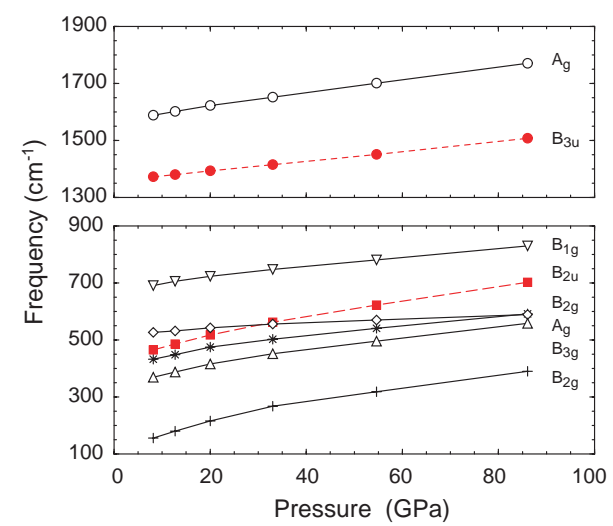

FIG. 4. Calculated zone-center phonons of $\mathrm{Cmcm}$ as a function of pressure. Modes having $\mathrm{B}_{2 u}$ and $\mathrm{B}_{3 u}$ symmetry are IR-active (red-dashed); the remaining are Raman-active (black-solid). Lines guide the eye.

Since there are two molecules in each primitive $\mathrm{Cmcm}$ cell, we expect nine optical phonons (plus three purely translational modes). At the zone-center, there are eight irreducible representations, as permitted by the $\mathrm{D}_{2 h}$ point group: four allow Raman activity $\left(\mathrm{A}_{g}, \mathrm{~B}_{1 g}\right.$, $\mathrm{B}_{2 g}$, and $\left.\mathrm{B}_{1 g}\right)$, three are IR-active $\left(\mathrm{B}_{1 u}, \mathrm{~B}_{2 u}\right.$, and $\left.\mathrm{B}_{3 u}\right)$, and a single remaining $\mathrm{A}_{u}$ mode is silent. Gorelli et al. [13, 15] have proposed a structure made up of two molecules (actually $\mathrm{O}_{4}$ units) having $\mathrm{D}_{2 h}$ symmetry. In agreement with Ref. 15], our predicted ground state possesses $\mathrm{D}_{2 h}$ point symmetry, but also includes additional lattice translations associated with the herringbone-type chain structure. Corresponding force constants are obtained through analysis [32] of a series of frozen-phonon calculations; phonon frequencies are then computed by diagonalization of block-diagonal dynamical matrices.

The pressure dependence of zone-center optical frequencies computed between 8 and $86 \mathrm{GPa}$ appears in Fig. 4 , and we obtain remarkably good agreement with the existing spectroscopic data, quite apart from the fact that $C 2 \mathrm{~mm}$ is favored above $47 \mathrm{GPa}$ in our calculations. Consistent with observations 15], we predict two IR-active modes over this range. The $\mathrm{B}_{3 u}$ mode is an antiphase vibron; its computed frequencies are in the 
range $1370-1480 \mathrm{~cm}^{-1}$, ywhich, while slightly lower than found in experiment [15], increase with increasing pressure in qualitative accord with measurements and a decreasing dimer length. The $\mathrm{B}_{2 u}$ mode is an antiphase libron-like mode with frequencies between $465-703 \mathrm{~cm}^{-1}$ over the pressure range 8-86 GPa. As with the vibron, these frequencies are somewhat larger than those found experimentally, which increase from 300 to $600 \mathrm{~cm}^{-1}$ between 10 and $70 \mathrm{GPa}$ at room temperature [14,15], a discrepancy that likely originates from the smaller $\mathrm{O}_{2}-\mathrm{O}_{2}$ distance we calculate. Since the decline in intermolecular distance associated with the $\mathrm{Cmcm}$ phase results in the formation of a permanent molecular polarization and intermolecular covalent bond, large dynamical effective charges (and hence absorptivity) would then be expected.

The Raman-active vibron $\left(\mathrm{A}_{g}\right)$ is in the range 1500$1650 \mathrm{~cm}^{-1}$, also in good agreement with measurement. Additionally, we predict five other Raman-active intermolecular modes, and two can ostensibly be assigned to observed peaks below $500 \mathrm{~cm}^{-1}$ 15.33, the $\mathrm{B}_{3 g}$ and lowest-frequency $\mathrm{B}_{2 g}$ modes. Since our calculations overestimate the IR-active mode frequencies, we regard this specific assignment as tentative. Extended scattering geometries with fully polarized radiation may be needed to elucidate three further Raman-active modes predicted here whose intensities may be weak, given the normal mode displacement patterns associated with the linear structure and given the very anisotropic molecular polarizability. In this context we note that the modes of interest are close in frequency to observed Raman peaks [33] which have been assigned, so far, to possible overtones and combinations of librons. It is also worth noting that even more modes would be expected of a more complicated $\epsilon$-phase, should it have a larger unit cell. Our results therefore suggest that an assessment of the phase diagram and lattice dynamics at low temperatures and over a wide pressure range will be of considerable experimental interest.

We gratefully acknowledge M. H. Cohen, M. P. Teter, and D. Vanderbilt for useful commentary; and we thank G. Kresse for providing the PAW potentials. This work was supported by the National Science Foundation (DMR-9988576). This work made use of the Cornell Center for Materials Research Shared Experimental Facilities, supported through the National Science Foundation Materials Research Science and Engineering Centers program (DMR-9632275).

[1] R. J. Meier and R. B. Helmholdt, Phys. Rev. B 29, 1387 (1984).

[2] M. Nicol and K. Syassen, Phys. Rev. B 28, 1201 (1983).

[3] D. Schiferl et al., Acta Crys. B 39, 153 (1983).

[4] Y. Akahama et al., Phys. Rev. Lett. 74, 4690 (1995).

[5] S. Desgreniers, Y. K. Vohra, and A. L. Ruoff, J. Phys. Chem. 94, 1117 (1990).
[6] M. Bastea, A. C. Mitchell, and W. J. Nellis, Phys. Rev. Lett. 86, 3108 (2001).

[7] K. Shimizu et al. Nature (London) 393, 767 (1998).

[8] M. F. Nicol, K. R. Hirsch, and W. B. Holzapfel, Chem. Phys. Lett. 68, 49 (1979).

[9] S. W. Johnson, M. Nicol, and D. Schiferl, J. Appl. Crys. 26, 320 (1993).

[10] S. Desgreniers and K. E. Brister, in High Pressure Science and Technology, W. A. Trzeciakowski, ed. (World Scientific, Singapore, 1996), 363.

[11] S. F. Agnew, B. I. Swanson, and L. H. Jones, J. Chem. Phys. 86, 5239 (1987).

[12] Y. Akahama, H. Kawamura, and O. Shimomura, Phys. Rev. B 64, 054105 (2001).

[13] F. A. Gorelli et al., Phys. Rev. Lett. 83, 4093 (1999).

[14] Y. Akahama and H. Kawamura, Phys. Rev. B 61, 8801 (2000).

[15] F. A. Gorelli et al., Phys. Rev. B 63, 104110 (2001).

[16] B. Edwards and N. W. Ashcroft, Nature (London) 388, 352 (1997).

[17] S. Serra et al., Phys. Rev. Lett. 80, 5160 (1998).

[18] P. Hohenberg and W. Kohn, Phys. Rev. 136, 864B (1964); W. Kohn and L. J. Sham, Phys. Rev. 140, 1133A (1965).

[19] J. P. Perdew et al., Phys. Rev. B 46, 6671 (1992); S. H. Vosko, L. Wilk, and M. Nusair, Can. J. Phys. 58, 1200 (1980).

[20] P. Blöchl, Phys. Rev. B 50, 17953 (1994); G. Kresse and D. Joubert, Phys. Rev. B 59, 1758 (1999).

[21] G. Kresse and J. Hafner, Phys. Rev. B 47, RC558 (1993); G. Kresse and J. Furthmüller, Comput. Mat. Sci. 6, 1550 (1996).

[22] J. P. Perdew, K. Burke, and M. Ernzerhof, Phys. Rev. Lett. 80, 891 (1998); F. W. Kutzler and G. S. Painter, Phys. Rev. B 45, 3236 (1992).

[23] Landolt-Börnstein: Numerical Data and Functional Relationships in Science and Technology, (eds. Hellwege, K.-H. and Olsen, J. L.), Group I, Vol. 3, 510 (SpringerVerlag, Berlin, 1951).

[24] See R. D. Etters, K. Kobashi, and J. Belak, Phys. Rev. B 32, 4097 (1985) and references therein.

[25] K. Rapcewicz and N. W. Ashcroft, Phys. Rev. B 44, 4032 (1991).

[26] W. Kohn, Y. Meir, and D. E. Makarov, Phys. Rev. Lett. 80, 4153 (1998).

[27] J. Yen and M. Nicol, J. Phys. Chem. 91, 3336 (1987).

[28] Relaxation of larger supercells containing up to 36 randomly-displaced molecules (constructed from 4molecule orthorhombic cells tripled along [100] and [010]) did not result in phases with lower enthalpy.

[29] Our predicted lattice parameters for antiferromagnetic Fmmm below $20 \mathrm{GPa}$ yield $b / a \sim 0.95$ and $c / a \sim 2$. Above $50 \mathrm{GPa}$ non-magnetic $C 2 \mathrm{~mm}$ is very nearly molecular close-packed, and $b / a \sim 0.58$ and $c / a \sim 1.67$.

[30] L. Hedin, Phys. Rev. 139, A796 (1965); M. S. Hybertsen and S. G. Louie, Phys. Rev. B 34, 5390 (1986).

[31] M. Santoro et al., Phys. Rev. B 64, 064428 (2001).

[32] SMODES was developed by H. T. Stokes and D. M. Hatch. See http://128.187.18.10/ stokesh/smodes.htm.

[33] Y. Akahama and H. Kawamura, Phys. Rev. B 54, R15602 (1996). 